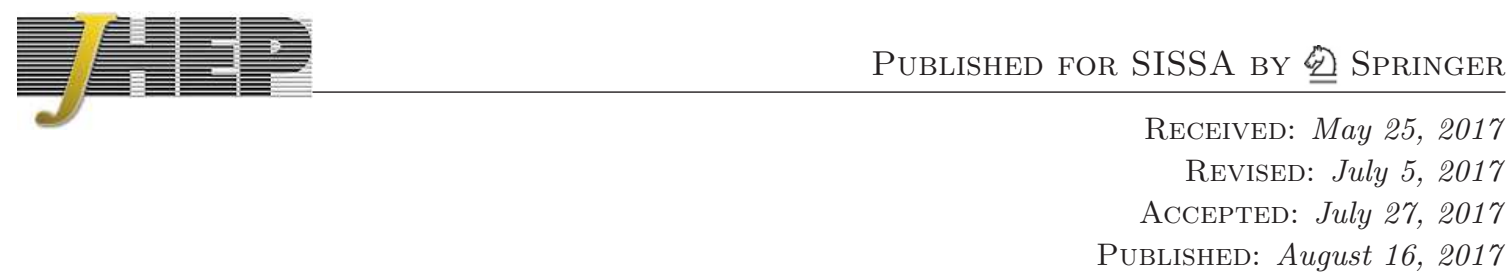

\title{
Anomaly-free dark matter models are not so simple
}

\author{
John Ellis, ${ }^{a, b}$ Malcolm Fairbairn ${ }^{a}$ and Patrick Tunney ${ }^{a}$ \\ ${ }^{a}$ Theoretical Particle Physics and Cosmology Group, Physics Department, King's College London, \\ London WC2R 2LS, U.K. \\ ${ }^{b}$ Theoretical Physics Department, CERN, \\ CH-1211 Geneva 23, Switzerland \\ E-mail: John.Ellis@cern.ch, malcolm.fairbairn@kcl.ac.uk, \\ patrick.tunney@kcl.ac.uk
}

ABSTRACT: We explore the anomaly-cancellation constraints on simplified dark matter (DM) models with an extra $\mathrm{U}(1)^{\prime}$ gauge boson $Z^{\prime}$. We show that, if the Standard Model (SM) fermions are supplemented by a single DM fermion $\chi$ that is a singlet of the SM gauge group, and the SM quarks have non-zero $\mathrm{U}(1)^{\prime}$ charges, the SM leptons must also have non-zero $\mathrm{U}(1)^{\prime}$ charges, in which case LHC searches impose strong constraints on the $Z^{\prime}$ mass. Moreover, the DM fermion $\chi$ must have a vector-like $\mathrm{U}(1)^{\prime}$ coupling. If one requires the $\mathrm{DM}$ particle to have a purely axial $\mathrm{U}(1)^{\prime}$ coupling, which would be the case if $\chi$ were a Majorana fermion and would reduce the impact of direct DM searches, the simplest possibility is that it is accompanied by one other new singlet fermion, but in this case the $\mathrm{U}(1)^{\prime}$ charges of the SM leptons still do not vanish. This is also true in a range of models with multiple new singlet fermions with identical charges. Searching for a leptophobic model, we then introduce extra fermions that transform non-trivially under the SM gauge group. We find several such models if the DM fermion is accompanied by two or more other new fermions with non-identical charges, which may have interesting experimental signatures. We present benchmark representatives of the various model classes we discuss.

Keywords: Anomalies in Field and String Theories, Beyond Standard Model

ARXIV EPRINT: 1704.03850 


\section{Contents}

1 Introduction 1

2 New SM singlet fermions and vanishing $\mathrm{U}(1)^{\prime}$ couplings to leptons? 4

3 A DM particle with axial $Z^{\prime}$ couplings?

4 New fermions transforming non-trivially under the SM gauge group $\quad 8$

$\begin{array}{lll}5 & \text { Summary } & 13\end{array}$

\section{Introduction}

The astrophysical and cosmological necessity for dark matter (DM) (see, for example, [1-10]) is one of the strongest motivations for particle physics beyond the Standard Model (SM). However, as yet there is no experimental evidence for any of the proposals for extensions of the SM, such as supersymmetry, that provide well-motivated models for DM particles [11-13]. Under these circumstances, a favoured approach is to model dark matter from the bottom up, in other words to avoid a priori theoretical assumptions and proceed phenomenologically.

Initially this programme began by considering higher-dimensional contact interactions $[14,15]$ where it is straightforward to compare constraints from collider production of dark matter with those from direct detection experiments [16]. Such toy models are very useful, but have obvious limitations, since unitarity inevitably breaks down at some scale. This may, on its own, not be viewed as being problematic in this entirely phenomenological approach, however often the features required to protect unitarity (for example new mediators) themselves lead to interesting phenomenology which is lost in the contact interaction setting [17-21]. The introduction of simplified dark matter models (SDMMs) with the minimal combination of features that a model of DM should have represents an attempt to address this in the simplest way possible [22-28]. Typically, these SDMMs contain, in addition to the DM particle itself that is often taken to be a fermion, $\chi$, and a bosonic intermediary, $Z^{\prime}$ (or $\phi$ ), that generates the interactions between $\chi$ and SM particles and prevents the inherent problems associated with the contact interaction.

There are then, in general, a number of free parameters associated with the model, for example the masses of the DM and intermediary particles and the seperate couplings of the intermediary to both the DM and SM particles. One then considers and combines the constraints on these parameters from laboratory experiments at accelerators such as the LHC, direct and indirect astrophysical searches for DM particles, and the allowed range of the cosmological DM density [29, 30]. These constraints depend, in particular, whether 
the DM particle $\chi$ is assumed to be Majorana or Dirac, whether the intermediary has spin zero or spin one, ${ }^{1}$ in which case it would be associated with an additional $\mathrm{U}(1)^{\prime}$ gauge symmetry, and whether the mediator couplings are scalar, pseudoscalar, vector or axial vector, all of which have different phenomenologies and constraints [23-25].

While these simple extensions of the SM are extremely useful for setting up a parameter space which can subsequently be explored, it is well known that many of the simplest models in the SDMM programme are not entirely self-consistent physically. For example, models with a massive gauge boson mediator do not respect unitarity to arbitrarily high scales unless set within a larger theory where the mass of that boson is explained through an additonal Brout-Englert-Higgs mechanism [31]. Introducing a dark Higgs sector can make such theories more palatable, but the presence of that sector can change the phenomenology of the model.

In this paper we focus on another issue, namely the fact that proposed SDMM extensions to the SM with spin-one mediators generally contain anomalies whose cancellation requires additional fermions. As pointed out in [31], the masses of the new fermions should be of the same order as the $\mathrm{U}(1)^{\prime}$ boson mass, offering additional LHC signatures that may already be constrained by the data and should be taken into account in constraining such not-so-SDMMs.

In the case of such a spin-one intermediary particle $Z^{\prime}$, renormalizability of the SDMM requires that it be free of anomalous triangle diagrams involving any combination of the SM gauge fields, the $\mathrm{U}(1)^{\prime}$ gauge field and the graviton [32]. ${ }^{2}$ The requirement of anomaly freedom is understood by constructors of SDMMs [31, 33], but in many cases its implications have not been pursued fully. One could, of course, take the point of view that any anomalies in the SDMM could be cancelled by some unspecified ultraviolet completion. However, in this paper we take the point of view that the SDMM should be self-consistent at the $\mathrm{U}(1)^{\prime}$ scale, so that one should try to construct anomaly-free SDMMs, and that it is interesting and important to understand what are the minimal such theories. ${ }^{3}$

There is a large literature on anomaly-free $\mathrm{U}(1)^{\prime}$ extensions of the SM with various motivations, see for example [33-52]. Among these, the closest in spirit to our paper are [42-46], and we comment later on the relations between their papers and ours. Typical extensions of the SM with a neutral $Z^{\prime}$ particle come from GUT theories and couple to leptons as well as quarks [53]. When such a particle acts as the mediator between the SM and a DM fermion, the two strongest constraints come from dilepton events at the LHC and direct detection experiments.

Models in which the $Z^{\prime}$ boson couples to leptons are very easy to constrain experimentally, since they yield dilepton events that give clear signals in hadron colliders without the backgrounds that dijets would experience, see for example [26]. Depending on the

\footnotetext{
${ }^{1}$ In principle, one could also consider models in which the mediator spin is $\geq 2$, but these have not yet found much favour.

${ }^{2}$ Studies of possible anomaly-free $\mathrm{U}(1)^{\prime}$ extensions of the SM started at least 40 years ago, though with different motivations. See, e.g., [32].

${ }^{3}$ The information gathered in this study may also help to guide intuition towards an ultraviolet-complete theory, if one adopts the alternative philosophy.
} 
model, lower bounds $m_{Z^{\prime}} \gtrsim 3 \mathrm{TeV}$ may be imposed by the LHC experiments [54, 55]. It therefore becomes important to try to suppress the coupling of the mediator particle to the SM leptons for couplings and masses that give rise to good relic abundance from thermal freeze-out. This is why one seeks SDMMs containing leptophobic vector mediators that couple only to quarks.

The second very tight constraint comes from the long reach of the latest direct detection experiments - at the time of writing the PandaX and LUX experiments have the leading sensitivity to spin-independent dark matter-nucleon scattering, and have reached cross sections as low as $10^{-46} \mathrm{~cm}^{2}$ for a DM particle mass of $30 \mathrm{GeV}[56,57]$. This makes it increasingly difficult to arrange couplings and mediator masses that give good relic abundance and are not ruled out, in the case of a vector mediator interaction that would generate coherent scattering on all the nucleons in the Xenon nucleus. This coherent scattering is suppressed by the relative particle velocity if the mediator has an axial coupling to dark matter, and additionally by momentum exchange if it has only axial couplings to quarks [58]. ${ }^{4}$

The following are the anomaly cancellation conditions involving the $\mathrm{U}(1)^{\prime}$ gauge field that are to be satisfied, ${ }^{5}$ where the trace is over all fermion species with non-trivial couplings to the corresponding gauge group factors:

(a) $\left[\mathrm{SU}(3)_{C}^{2}\right] \times\left[\mathrm{U}(1)^{\prime}\right]$, which implies $\operatorname{Tr}\left[\left\{\mathcal{T}^{i}, \mathcal{T}^{j}\right\} Y^{\prime}\right]=0$.

(b) $\quad\left[\mathrm{SU}(2)_{W}^{2}\right] \times\left[\mathrm{U}(1)^{\prime}\right]$, which implies $\operatorname{Tr}\left[\left\{T^{i}, T^{j}\right\} Y^{\prime}\right]=0$.

(c) $\left[\mathrm{U}(1)_{Y}^{2}\right] \times\left[\mathrm{U}(1)^{\prime}\right]$, which implies $\operatorname{Tr}\left[\mathrm{Y}^{2} Y^{\prime}\right]=0$.

(d) $\left[\mathrm{U}(1)_{Y}\right] \times\left[\mathrm{U}(1)^{\prime^{2}}\right]$, which implies $\operatorname{Tr}\left[Y Y^{\prime 2}\right]=0$.

(e) $\left[\mathrm{U}(1)^{\prime 3}\right]$, which implies $\operatorname{Tr}\left[Y^{\prime 3}\right]=0$.

(f) Gauge-gravity, which implies $\operatorname{Tr}[Y]=\operatorname{Tr}\left[Y^{\prime}\right]=0$.

As we shall see, satisfying these conditions with the DM fermion $\chi$ being the only fermion beyond the SM requires that the $\mathrm{U}(1)^{\prime}$ boson couples to both leptons and quarks, exposing it to sensitive LHC searches, and that the DM fermion has vector-like $Z^{\prime}$ couplings, placing it within reach of direct searches for DM scattering. A purely axial $\chi-Z^{\prime}$ coupling is possible only if there are additional new fermions. The intermediary boson would still have $\mathrm{U}(1)^{\prime}$ couplings to leptons as well as quarks if there is just one extra singlet fermion, and in a range of models with multiple new singlet fermions with identical charges. Continuing the search for a model with vanishing lepton couplings, we then consider models with extra fermions transforming non-trivially as doublets or triplets of $\mathrm{SU}(2)_{W}$ as well as singlets. We find several classes of such models if the DM fermion is accompanied by two or more other new fermions with non-identical charges, generalizing a model presented in [42-45].

\footnotetext{
${ }^{4}$ However, we caution that renormalization effects below the $\mathrm{U}(1)^{\prime}$ mass scale may enhance significantly the scattering of an axially-coupled DM fermion [59].

${ }^{5}$ We follow the notation in [33]: $\mathcal{T}^{i}$ is a generator of $\mathrm{SU}(3)_{C}, T^{i}$ is a generator of $\mathrm{SU}(2)_{W}$ and $Y, Y^{\prime}$ are hypercharge and $\mathrm{U}(1)^{\prime}$ charge matrices respectively. The $\mathrm{U}(1)$ charge matrices are proportional to the identity, but taking the trace will give a factor of two for a doublet relative to a singlet, for example.
} 


\section{New SM singlet fermions and vanishing $\mathrm{U}(1)^{\prime}$ couplings to leptons?}

We consider first the possibility that the SDMM contains extra fermions that are singlets under the SM gauge group. We assume also that the different quark and lepton generations have identical $\mathrm{U}(1)^{\prime}$ charges, so as to minimize flavour-changing neutral currents. In this case, the anomaly-cancellation conditions above take the forms [37]:

$$
\begin{aligned}
& \text { (a) } 3\left(2 Y_{q}^{\prime}-Y_{u}^{\prime}-Y_{d}^{\prime}\right)=0, \\
& \text { (b) } 9 Y_{q}^{\prime}+3 Y_{l}^{\prime}=0, \\
& \text { (c) } 2 Y_{q}^{\prime}-16 Y_{u}^{\prime}-4 Y_{d}^{\prime}+6\left(Y_{l}^{\prime}-2 Y_{e}^{\prime}\right)=0, \\
& \text { (d) } 6\left(Y_{q}^{\prime 2}-2 Y_{u}^{\prime 2}+Y_{d}^{\prime 2}\right)-6\left(Y_{l}^{\prime 2}-Y_{e}^{\prime 2}\right)=0, \\
& \text { (e) } 9\left(2 Y_{q}^{\prime 3}-Y_{u}^{\prime 3}-Y_{d}^{\prime 3}\right)+3\left(2 Y_{l}^{\prime 3}-Y_{e}^{\prime 3}\right)+\operatorname{Tr}_{\mathrm{BSM}}\left(Y^{\prime 3}\right)=0, \\
& \text { (f) } 9\left(2 Y_{q}^{\prime}-Y_{u}^{\prime}-Y_{d}^{\prime}\right)+3\left(2 Y_{l}^{\prime}-Y_{e}^{\prime}\right)+\operatorname{Tr}_{\mathrm{BSM}}\left(Y^{\prime}\right)=0 .
\end{aligned}
$$

where the fermionic $\mathrm{U}(1)^{\prime}$ charges are denoted by $Y_{i}^{\prime}, q$ and $l$ label the left-handed quark and lepton doublets, the right-handed fields are labelled $u, d, e$, and $\operatorname{Tr}_{\mathrm{BSM}}$ denotes a trace over the additional fermions beyond the SM. ${ }^{6}$

In the absence of BSM particles, the anomaly cancellation conditions depend only on the $Y^{\prime}$ charges of the SM fields. The Y-sequential model $[33,36]$ is a well known example of an anomaly-free $\mathrm{U}(1)^{\prime}$ theory where the $Y^{\prime}$ charge of each fermion is proportional to the $\mathrm{SM}$ $Y$ hypercharge. This solution is trivially guaranteed to exist since the SM is anomaly-free, and so we expect to recover this model in our analysis when $\operatorname{Tr}_{\mathrm{BSM}}\left(Y^{\prime}\right)=\operatorname{Tr}_{\mathrm{BSM}}\left(Y^{\prime 3}\right)=0$. However, this model has couplings to leptons and hence is subject to the strong LHC dilepton constraints, so first we will see if it is possible to obtain an anomaly-free theory with vanishing couplings to leptons.

In addition to these anomaly cancellation conditions, gauge invariance of the SM Yukawa interactions require, if there is a single Higgs doublet,

$$
Y_{H}^{\prime}=Y_{q}^{\prime}-Y_{u}^{\prime}=Y_{d}^{\prime}-Y_{q}^{\prime}=Y_{e}^{\prime}-Y_{l}^{\prime},
$$

where $Y_{H}^{\prime}$ is the $\mathrm{U}(1)^{\prime}$ charge of the SM Higgs. ${ }^{7}$ These relations always ensure that the first anomaly condition is satisfied, motivating the consideration of new fermions that are $\mathrm{SU}(3)$ singlets as the simplest possibility. If one does not want to assume a particular mass generation mechanism for the SM fields, we note that equation (2.7) is redundant when equations (2.1)-(2.3) are solved with exotic fermions transforming trivially with respect to the SM gauge group. As such, our conclusions in this section and in section 3 are independent of the Yukawa sector, but we impose (2.7) as independent constraints in section 4 .

\footnotetext{
${ }^{6}$ The anomaly-cancellation conditions for the model studied in [42-45] are more complicated, as it has 2 extra $\mathrm{U}(1)$ gauge factors, corresponding to baryon and lepton number $B$ and $L$. However, in the limit where one discards the $\mathrm{U}(1)_{L}$ boson it becomes a leptophobic model with a single $\mathrm{U}(1)^{\prime}$ equivalent to $\mathrm{U}(1)_{B}$, as we discuss later.

${ }^{7}$ The conditions (2.7) were not imposed in the models studied in [46], which would require multiple Higgs representations in order to accommodate SM fermion masses and quark mixing.
} 
We focus first on the second anomaly condition (2.2) that involves $\mathrm{SU}(2)_{W}$ gauge fields, which we rewrite as:

$$
Y_{l}^{\prime}=-3 Y_{q}^{\prime}
$$

This equation implies directly that if $Y_{l}^{\prime}=0$, so as to avoid the strong constraints from dilepton searches at the LHC, then also $Y_{q}^{\prime}=0$. We then consider the second Yukawa condition in (2.7), namely $Y_{d}^{\prime}-Y_{q}^{\prime}=Y_{e}^{\prime}-Y_{l}^{\prime}$. If we now require that $Y_{e}^{\prime}=0$, again so as to avoid the LHC dilepton constraints, we see that also $Y_{d}^{\prime}=0$ and hence, via the first anomaly condition (2.1), also $Y_{u}^{\prime}=0$. We conclude that the boson of a $\mathrm{U}(1)^{\prime}$ model designed to avoid the LHC dilepton constraints would not even be produced via tree-level quark-antiquark annihilations at the LHC.

Moreover, we note that, if the DM particle $\chi$ is the only new fermion, the fifth and sixth anomaly conditions (2.5), (2.6) require

$$
\begin{gathered}
3\left(Y_{u}^{\prime}-4 Y_{q}^{\prime}\right)^{3}+Y_{\chi, L}^{\prime 3}-Y_{\chi, R}^{\prime 3}=0 \\
3\left(Y_{u}^{\prime}-4 Y_{q}^{\prime}\right)+Y_{\chi, L}^{\prime}-Y_{\chi, R}^{\prime}=0,
\end{gathered}
$$

to which the only rational solution is $Y_{\chi, L}^{\prime}=Y_{\chi, R}^{\prime}$ implying that such a 'singleton' DM particle must have a vector-like $\mathrm{U}(1)^{\prime}$ coupling, but not constraining its magnitude. This solution also implies from (2.10) that $Y_{u}^{\prime}=4 Y_{q}^{\prime}$.

To summarize this section, assuming that the $\mathrm{U}(1)^{\prime}$ charges of the SM fermions are generation-independent, and that any new fermions that are chirally charged under $\mathrm{U}(1)^{\prime}$ are singlets under the SM gauge group, we found that the intermediary $\mathrm{U}(1)^{\prime}$ boson must have leptonic couplings and hence be subject to LHC searches for dilepton signatures. Moreover, if the DM particle is the only such new singlet fermion, it must have a vector-like $\mathrm{U}(1)^{\prime}$ coupling. This would also be the case if there were other new SM-singlet fermions that are vectorial under $\mathrm{U}(1)^{\prime}$, since they would not contribute to any of the anomaly equations (2.1) to (2.6). This benchmark model has two ${ }^{8}$ free coupling parameters, $Y_{\chi, L}^{\prime}=$ $Y_{\chi, R}^{\prime}$ and $Y_{q}^{\prime}$, in terms of which the $Z^{\prime}$ couplings of the other SM fermions and the SM Higgs boson are specified as follows:

$$
Y_{l}^{\prime}=-3 Y_{q}^{\prime}, \quad Y_{e}^{\prime}=-6 Y_{q}^{\prime}, \quad Y_{d}^{\prime}=-2 Y_{q}^{\prime}, \quad Y_{u}^{\prime}=4 Y_{q}^{\prime}, \quad Y_{H}^{\prime}=-3 Y_{q}^{\prime} .
$$

It is possible to scale the overall couplings of the SM and dark sector to the $Z^{\prime}$ independently, although creating a large hierarchy would require accepting the same hierarchy between $\mathrm{U}(1)^{\prime}$ charges.

\section{A DM particle with axial $Z^{\prime}$ couplings?}

We now study whether the DM fermion could have an axial $Z^{\prime}$ coupling if we allow more new SM-singlet fermions that possess only $\mathrm{U}(1)^{\prime}$ charges, in which case the constraints from experiments searching directly for DM scattering would be weaker [59]. We also recall that an axial $\mathrm{U}(1)^{\prime}$ is the only possibility if the DM particle is a Majorana fermion.

\footnotetext{
${ }^{8}$ However, choosing to normalise one of the $\mathrm{Y}^{\prime}$ charges with the freedom to rescale the dark gauge coupling would leave only one free parameter.
} 
The constraints (2.7) and (2.8) remain valid in this case, so the anomaly conditions (2.1) to (2.4) are all satisfied automatically, and we need only consider the remaining conditions (2.5), (2.6), which we write in the forms

$$
\begin{gathered}
3\left(Y_{u}^{\prime}-4 Y_{q}^{\prime}\right)^{3}+\sum_{j}\left(Y_{j, L}^{\prime 3}-Y_{j, R}^{\prime 3}\right)=0, \\
3\left(Y_{u}^{\prime}-4 Y_{q}^{\prime}\right)+\sum_{j}\left(Y_{j, L}^{\prime}-Y_{j, R}^{\prime}\right)=0,
\end{gathered}
$$

where $Y_{j, L / R}^{\prime}$ is the $\mathrm{U}(1)^{\prime}$ charge of the left/right-handed component of a new fermion species $j$.

One obvious solution has $Y_{u}^{\prime}=4 Y_{q}^{\prime}$ and any number of new fermions with $Y_{j, L}^{\prime}=Y_{j, R}^{\prime}$. In the case of a single new fermion (presumably the DM particle) this is in fact the only solution, as discussed in the previous section. It is clear from equations (3.1), (3.2) that if we require a purely axial $Z^{\prime}$ coupling of the new DM fermion $\chi$, we will need at least one other fermion that is charged under $\mathrm{U}(1)^{\prime}$ in order to cancel the DM anomaly contributions.

Therefore, we consider now models that, in addition to a candidate DM particle $\chi$ with charge $Y_{\chi, L}^{\prime}=-Y_{\chi, R}^{\prime}$, contain a single other species $A$ with left- and right-handed charges $Y_{A, L}^{\prime}$ and $Y_{A, R}^{\prime}$ under $\mathrm{U}(1)^{\prime}$ that is also a singlet under the SM group. Solving equations (3.1) and (3.2) above, we find that that this last equation can be written as

$$
Y_{u}^{\prime}=4 Y_{q}^{\prime}-\frac{1}{3}\left(Y_{A, L}^{\prime}-Y_{A, R}^{\prime}\right)-\frac{2}{3} Y_{\chi, L}^{\prime}
$$

Substituting this condition into equation (3.1) gives a relatively complicated polynomial equation. Using the arbitrary normalization $Y_{\chi, L}^{\prime}=1$, the solutions we find with $\mathrm{U}(1)^{\prime}$ charges that are the smallest rational numbers are

$$
\begin{aligned}
& Y_{A, L}^{\prime}=-1, \quad Y_{A, R}^{\prime}=1, \\
& Y_{A, L}^{\prime}=0, \quad Y_{A, R}^{\prime}=-1 \quad \text { or } \quad Y_{A, R}^{\prime}=5 / 4 \text {, } \\
& Y_{A, R}^{\prime}=0, \quad Y_{A, L}^{\prime}=-5 / 4 \quad \text { or } \quad Y_{A, L}^{\prime}=1 \text {, }
\end{aligned}
$$

where the last pairs of solutions are equivalent, being mirror images.

In general, there will be mixing between the new neutral fermions $(\chi, A)$ induced by a combination of 'Majorana' mass terms that do not require $\mathrm{U}(1)^{\prime}$ breaking and 'Dirac' terms that involve the intervention of a Higgs vacuum expectation value (vev). As a result, the mass eigenstates will be orthogonal mixtures of the interaction eigenstates, and the lightest one should be identified as the DM particle. The pattern of mixing is quite modeldependent, being determined by the assumed pattern of Majorana-type masses that do not require a Higgs vev as well as the assumed set of Higgs representations, their vev's and the magnitudes of their couplings. For example, in model (3.4) above, there could be a $2 \times 2$ Majorana-type mass matrix for the $\chi_{L}$ and $\overline{\chi_{R}}$, and Dirac terms due to a Higgs with $Y_{H}^{\prime}=2$ could extend this to a full rank-4 mass matrix for $\chi_{L}, \overline{\chi_{R}}, A_{L}$ and $\overline{A_{R}}$. On the other hand, generating a full rank-4 mass matrix in the first model in (3.5) or the second model in (3.6) would also require a Higgs with $Y_{H}^{\prime}=1$, and obtaining a rank-4 matrix matrix in the other models in (3.5) and (3.6) would require also Higgs fields with fractional $Y^{\prime}$. 
Since the fermion species in the dark sector have different $\mathrm{U}(1)^{\prime}$ charges, they do not respect the Glashow-Weinberg-Paschos conditions for natural flavour conservation [60, 61], and the $Z^{\prime}$ will in general have off-diagonal interactions with the dark mass eigenstates. The heavier mass eigenstates could therefore decay into the DM particle by radiating SM $\bar{f} f$ pairs through a virtual $Z^{\prime} .{ }^{9}$ We have identified this DM particle with the $\chi$ interaction eigenstate introduced above, which would indeed be the lightest mass eigenstate in a suitable degenerate limit of the mass matrix. In this limit it would have a purely axial $\mathrm{U}(1)^{\prime}$ coupling, and this would also be the case for arbitrary mixing in model (3.4), where both $\chi$ and $A$ have axial couplings. However, in the cases (3.6) the coupling of the lightest mass eigenstate would not be purely axial if the mixing were non-trivial.

We have searched for all other solutions with rational $\mathrm{U}(1)^{\prime}$ charges of the form $p / q$ : $|p, q| \in \mathbb{Z}$ and $\leq 100$, with the following results

$$
\begin{aligned}
Y_{A, L}^{\prime} & =2, & Y_{A, R}^{\prime} & =-\frac{1}{2}, \\
Y_{A, L}^{\prime} & =-\frac{8}{5}, & Y_{A, R}^{\prime} & =-\frac{7}{5}, \\
Y_{A, L}^{\prime} & =\frac{25}{9}, & Y_{A, R}^{\prime} & =-\frac{29}{9}
\end{aligned}
$$

and equivalent mirror solutions. However, in all these cases the SM leptons have non-zero $\mathrm{U}(1)^{\prime}$ charges.

We have also explored the possibilities for two or three 'generations' of new fermions $X, A$ with 'generation'-independent charges. In both cases the first solution in (3.6) is again valid, and in the three-'generation' case there is in addition a solution with $Y_{A, L}^{\prime}=$ $0, Y_{A, R}^{\prime}=1$ and its mirror. We have not studied the two- and three-'generation' case thoroughly but there are, in general, fewer solutions within any fixed range of $p$ and $q$ than in the single-'generation' case (3.6), (3.7), and the SM leptons again have non-zero U(1)' charges.

We conclude that, if the DM particle is required to have an axial $\mathrm{U}(1)^{\prime}$ charge so as to minimize the impacts of DM search experiments, not only will the $\mathrm{U}(1)^{\prime}$ gauge boson again have leptonic couplings, but also there must be additional fermions with $\mathrm{U}(1)^{\prime}$ charges that could be produced and detected at the LHC. The simplest solutions have the following $\mathrm{U}(1)^{\prime}$ charges (using the normalization $Y_{\chi, L}^{\prime}=-Y_{\chi, R}^{\prime}=1$ ):

$$
\begin{array}{ll}
Y_{A, L}^{\prime}=-1, & Y_{A, R}^{\prime}=1 . \\
Y_{A, L}^{\prime}=0, & Y_{A, R}^{\prime}=-1
\end{array} \quad\left(\text { or } \quad Y_{A, R}^{\prime}=0, Y_{A, L}^{\prime}=1\right) .
$$

These two models also have $Y_{q}^{\prime}$ as a free parameter, and the remaining SM U(1)' charges

\footnotetext{
${ }^{9}$ This suggests the possibility of an LHC signature that complements the familiar monojet/photon/Higgs... searches, namely one in which an on-shell $Z^{\prime}$ is produced and decays into the DM particle and a heavier dark particle whose decay yields a missing energy + dijet final state. In this case there may be no need to require any initial-state boson radiation.
} 
are then related (for both models) by the following equation

$$
\begin{array}{ll}
Y_{l}^{\prime}=-3 Y_{q}^{\prime}, & Y_{u}^{\prime}=4 Y_{q}^{\prime}-\frac{1}{3}\left(Y_{A, L}^{\prime}-Y_{A, R}^{\prime}\right)-\frac{2}{3} Y_{\chi, L}^{\prime}, \quad Y_{d}^{\prime}=2 Y_{q}^{\prime}-Y_{u}^{\prime}, \\
Y_{e}^{\prime}=-2 Y_{q}^{\prime}-Y_{u}^{\prime}, & Y_{H}^{\prime}=Y_{q}^{\prime}-Y_{u}^{\prime} .
\end{array}
$$

DM searches at the LHC are often presented in a way that shows the complementarity between the production of DM and resonant searches for the mediator, for example when comparing missing energy and dijet searches. This presentation is only possible if one is able to treat the dark and visible couplings as independent parameters, which would be possible for (3.8) but not (3.9). This is because the anomaly cancellation in model (3.8) occurs independently in the dark and visible sectors. This allows the dark and SM couplings of the fermions to the $Z^{\prime}$ to be scaled independently, with the caveat that one would have to be prepared to accept very large or very small charges in order to create a large hierarchy between the dark and visible couplings. On the other hand, anomaly cancellation in model (3.9) relates directly the charges of the dark and visible sectors.

Finally, we recall that only in case (3.8) is the DM particle coupling guaranteed to be purely axial, whatever the amount of dark fermion mixing.

\section{New fermions transforming non-trivially under the SM gauge group}

In this section we introduce exotic fermions to cancel the anomalies present in a leptophobic theory. We first build up the minimal field content needed to obtain an anomaly-free solution, before commenting on whether there is still a viable DM candidate $\chi$ present in the theory.

We consider the possibility that there are new fermions transforming under non-trivial representations of the SM gauge group,${ }^{10}$ in which case the question of whether the leptonic $\mathrm{U}(1)^{\prime}$ charges vanish is reopened. In such a case one would also need to ensure the cancellation of the anomalies involving only SM gauge bosons, which are not listed above. These SM anomalies would vanish if the fermions are vector-like with respect to the SM gauge group, and then the new fermions would contribute only to the anomalies listed above if they are chiral with respect to the $\mathrm{U}(1)^{\prime}$. This option would open up possibilities for other electroweak signatures, if they are not too heavy.

In order to analyse this possibility, we first repeat the anomaly conditions (2.1) to (2.6) above, using the Yukawa conditions (2.7) to substitute $Y_{u}^{\prime}$ and $Y_{d}^{\prime}$, and assuming that any new fermions transform in either the trivial or fundamental representations:

$$
\begin{array}{r}
\sum_{f \in \mathrm{SU}(3)}\left(Y_{f, L}^{\prime}-Y_{f, R}^{\prime}\right)=0, \\
3 Y_{l}^{\prime}+9 Y_{q}^{\prime}+\sum_{f \in \mathrm{SU}(2)}\left(Y_{f, L}^{\prime}-Y_{f, R}^{\prime}\right)=0, \\
-6\left(Y_{l}^{\prime}+3 Y_{q}^{\prime}\right)+\sum_{f}\left(Y_{f, L}^{\prime} Y_{f, L}^{2}-Y_{f, R}^{\prime} Y_{f, R}^{2}\right)=0,
\end{array}
$$

\footnotetext{
${ }^{10}$ The models studied in [42-46] all incorporate fermions that are charged under the $\mathrm{SM} \mathrm{SU}(3) \times \mathrm{SU}(2)$.
} 


$$
\begin{aligned}
12\left(Y_{e}^{\prime}-Y_{l}^{\prime}\right)\left(Y_{l}^{\prime}+3 Y_{q}^{\prime}\right)+\sum_{f}\left(Y_{f, L}^{\prime 2} Y_{f, L}-Y_{f, R}^{\prime 2} Y_{f, R}\right) & =0 \\
-3\left(Y_{e}^{\prime 3}-2 Y_{l}^{\prime 2}\left(Y_{l}^{\prime}-9 Y_{q}^{\prime}\right)+18 Y_{e}^{\prime 2} Y_{q}^{\prime}-36 Y_{e}^{\prime} Y_{l}^{\prime} Y_{q}^{\prime}\right)+\sum_{f}\left(Y_{f, L}^{\prime 3}-Y_{f, R}^{\prime 3}\right) & =0 \\
-3 Y_{e}^{\prime}+6 Y_{l}^{\prime}+\sum_{f}\left(Y_{f, L}^{\prime}-Y_{f, R}^{\prime}\right) & =0
\end{aligned}
$$

The simplest possibility we study is a single new fermion species $A$ that transforms in the fundamental of $\mathrm{SU}(2)$ and has both $\mathrm{U}(1)_{Y}$ and $\mathrm{U}(1)^{\prime}$ charges. In order not to mess up the purely SM anomaly conditions, we assume it is vector-like under both $\mathrm{SU}(2)_{W}$ and $\mathrm{U}(1)_{Y}$, so that $Y_{A, L}=Y_{A, R}=Y_{A}$. In this case the second and third anomaly cancellation conditions (4.2), (4.3) take the form

$$
\begin{array}{r}
3 Y_{l}^{\prime}+9 Y_{q}^{\prime}+Y_{A, L}^{\prime}-Y_{A, R}^{\prime}=0, \\
-6\left(Y_{l}^{\prime}+3 Y_{q}^{\prime}\right)+2 Y_{A}^{2}\left(Y_{A, L}^{\prime}-Y_{A, R}^{\prime}\right)=0 .
\end{array}
$$

Eliminating $Y_{q}^{\prime}$ by substituting (4.7) into (4.8), we find

$$
\left(1+Y_{A}^{2}\right)\left(Y_{A, L}^{\prime}-Y_{A, R}^{\prime}\right)=0,
$$

which has has only the vector-like solution $Y_{A, L}^{\prime}=Y_{A, R}^{\prime}$. Moreover, in this case $Y_{l}^{\prime}+3 Y_{q}^{\prime}=0$, so that $Y_{l}^{\prime}=0$ would require $Y_{q}^{\prime}=0$. Implementing full leptophobia by requiring $Y_{e}^{\prime}=0$ would then require the SM Higgs to have $Y_{H}^{\prime}=0$ and hence also $Y_{u}^{\prime}=Y_{d}^{\prime}=0$, again entailing vanishing couplings to quarks. The same conclusions hold for models with several new fermion 'generations' if their charges are 'generation'-independent, or if we had put $A$ in the adjoint representation.

We are therefore led to consider adding another new fermion species $B$ with different SM quantum numbers, imposing $Y_{l}^{\prime}=Y_{e}^{\prime}=0$ in the attempt to find a non-trivial leptophobic solution. If $A$ and $B$ are both doublets (or both triplets) under $\mathrm{SU}(2)$, the only solution is the one with all SM field charges vanishing. Therefore we consider the possibility that $A$ is a doublet under $\mathrm{SU}(2)_{W}$ but $B$ is an $\mathrm{SU}(2)_{W}$ singlet. In this case the second anomaly (4.2) gives $Y_{q}^{\prime}=-\frac{1}{9}\left(Y_{A, L}^{\prime}-Y_{A, R}^{\prime}\right)$ and the sixth anomaly (4.6) gives $Y_{B, R}^{\prime}=Y_{B, L}^{\prime}+2 Y_{A, L}^{\prime}-2 Y_{A, R}^{\prime}$. Substituting these into the third anomaly (4.3) yields

$$
\left(1+Y_{A}^{2}-Y_{B}^{2}\right)\left(Y_{A, L}^{\prime}-Y_{A, R}^{\prime}\right)=0
$$

We ignore the solution $Y_{A, L}^{\prime}=Y_{A, R}^{\prime}$ since it would imply $Y_{q}^{\prime}=0$, which would then make all SM charges vanish. Therefore we must require

$$
1+Y_{A}^{2}-Y_{B}^{2}=0
$$

to which the only integer solution is $\{0,1\}$. Since we are working in the convention where $Q=T^{3}+Y / 2$, this solution has half-integer electric charges for both $A$ and $B$, conflicting with the integer charge quantization seen in Nature [62]. We conclude that this solution is not acceptable. 
We have also looked for solutions where $A$ is an $\mathrm{SU}(2)_{W}$ triplet. Equation (4.2) is modified, as we are no longer considering fermions solely in the fundamental or trivial representation, becoming

$$
9 Y_{q}^{\prime}+\sum_{f \in \mathbf{2}}\left(Y_{f, L}^{\prime}-Y_{f, R}^{\prime}\right)+4 \sum_{f \in \mathbf{3}}\left(Y_{f, L}^{\prime}-Y_{f, R}^{\prime}\right)=0
$$

where $\mathbf{2}$ and $\mathbf{3}$ label the fundamental and adjoint representations respectively. If $B$ is again an $\mathrm{SU}(2)_{W}$ singlet, repeating the same steps as before we find the condition

$$
8+3 Y_{A}^{2}-3 Y_{B}^{2}=0
$$

which has no integer solutions. Finally, in the case where $A$ is a triplet and $B$ is a doublet we obtain the condition

$$
5+3 Y_{A}^{2}-3 Y_{B}^{2}=0,
$$

which also has no integer solutions. Moreover, we have checked that there are still no solutions in these triplet/singlet and triplet/doublet cases when there are several 'generations' of $A$ and $B$ (even with different numbers of each), as long as the $\mathrm{U}(1)^{\prime}$ charges are 'generation'-independent.

We are therefore led to consider models with three or more species of new fermions. The models studied in [42-46] all feature six new fermion species. However, as already commented, when the $\mathrm{U}(1)_{L}$ is discarded along with its three $\nu_{R}$ species, the model studied in [42-45] becomes a leptophobic model with a single $\mathrm{U}(1)^{\prime}$ that is equivalent to $\mathrm{U}(1)_{B}$. In this limit, the new fermions in the model comprise a doublet that is vector-like under $\mathrm{SU}(2)$ and has $Y=-1$, and two singlets with $Y=-2,0$, respectively. ${ }^{11}$

We have checked the anomaly-cancellation conditions for other models containing three new fermion species with different $\mathrm{U}(1)_{Y}$ charges, i.e., $A, B, \chi$ in the $\left(\mathrm{SU}(2)_{W}, \mathrm{U}(1)_{Y}\right.$, $\left.\mathrm{U}(1)_{Y^{\prime}}\right)$ representations $\left(2, \mathrm{Y}_{A}, \mathrm{Y}_{A, L / R}^{\prime}\right),\left(1, \mathrm{Y}_{B}, \mathrm{Y}_{B, L / R}^{\prime}\right)$, and $\left(1,0, \mathrm{Y}_{\chi, L / R}^{\prime}\right)$ respectively. In order to obtain a leptophobic solution with $Y_{l}^{\prime}=Y_{e}^{\prime}=0$, the SM Yukawa condition (2.7) imposes $Y_{u}^{\prime}=Y_{d}^{\prime}=Y_{q}^{\prime}$, so we choose $Y_{q}^{\prime}$ as the only remaining free SM charge. Normalizing $\mathrm{Y}_{\chi, L}^{\prime}=1$, and noting that the $\mathrm{SU}(3)$ anomaly condition is satisfied automatically when the Higgs coupling constraint (2.7) is imposed, the next four anomaly-cancellation conditions yield

$$
\begin{aligned}
Y_{q}^{\prime} & =\frac{1}{9}\left(Y_{A, R}^{\prime}-Y_{A, L}^{\prime}\right) \\
Y_{\chi, R}^{\prime} & =\frac{Y_{B}^{2}\left(2 Y_{A, L}^{\prime}-2 Y_{A, R}^{\prime}+1\right)+2\left(Y_{A}^{2}+1\right)\left(Y_{A, R}^{\prime}-Y_{A, L}^{\prime}\right)}{Y_{B}^{2}}, \\
Y_{B, L}^{\prime} & =\frac{\left(Y_{A} Y_{B}^{3}-2\left(Y_{A}^{2}+1\right)^{2}\right) Y_{A, L}^{\prime}+\left(Y_{A} Y_{B}^{3}+2\left(Y_{A}^{2}+1\right)^{2}\right) Y_{A, R}^{\prime}}{2\left(Y_{A}^{2}+1\right) Y_{B}^{2}}, \\
Y_{B, R}^{\prime} & =\frac{\left(Y_{A} Y_{B}^{3}+2\left(Y_{A}^{2}+1\right)^{2}\right) Y_{A, L}^{\prime}+\left(Y_{A} Y_{B}^{3}-2\left(Y_{A}^{2}+1\right)^{2}\right) Y_{A, R}^{\prime}}{2\left(Y_{A}^{2}+1\right) Y_{B}^{2}} .
\end{aligned}
$$

\footnotetext{
${ }^{11}$ In our convention of $Q=T_{3}+Y / 2$, the SM hypercharges are twice those in [42-45].
} 
Using these expressions, the final $\mathrm{U}(1)^{3}$ anomaly condition gives rise to the slightly unwieldy expression:

$$
\begin{aligned}
& -\frac{1}{8\left(Y_{A}^{2}+1\right)^{3} Y_{B}^{6}}\left[-16\left(Y_{A}^{2}+1\right)^{3} Y_{B}^{6}\left(\left(Y^{\prime}\right)_{A, L}^{3}-\left(Y^{\prime}\right)_{A, R}^{3}\right)\right. \\
& \quad+\left(\left(Y_{A} Y_{B}^{3}+2\left(Y_{A}^{2}+1\right)^{2}\right) Y_{A, L}^{\prime}+\left(Y_{A} Y_{B}^{3}-2\left(Y_{A}^{2}+1\right)^{2}\right) Y_{A, R}^{\prime}\right)^{3} \\
& \quad+\left(\left(2\left(Y_{A}^{2}+1\right)^{2}-Y_{A} Y_{B}^{3}\right) Y_{A, L}^{\prime}-\left(Y_{A} Y_{B}^{3}+2\left(Y_{A}^{2}+1\right)^{2}\right) Y_{A, R}^{\prime}\right)^{3} \\
& \quad+8\left(Y_{A}^{2}+1\right)^{3}\left(Y_{B}^{2}\left(2 Y_{A, L}^{\prime}-2 Y_{A, R}^{\prime}+1\right)+2\left(Y_{A}^{2}+1\right)\left(Y_{A, R}^{\prime}-Y_{A, L}^{\prime}\right)\right)^{3} \\
& \left.\quad-8\left(Y_{A}^{2}+1\right)^{3} Y_{B}^{6}\right]=0 .
\end{aligned}
$$

This equation has a symmetry $Y_{A / B} \leftrightarrow-Y_{A / B}$, which facilitates a scan of possible solutions. We have restricted our search to positive integer values $\leq 10$ for $Y_{A / B}$. The other unknowns, $Y_{A, L / R}^{\prime}$, are both rational, and we have scanned irreducible rational numbers of the form $\pm p / q$ with $p$ and $q$ integers $\leq 10$. In order to have integer charge quantisation, and recalling that our convention is $Q=T_{3}+Y / 2$, we further require $Y_{A}$ to be odd (since its a doublet) and $Y_{B}$ to be even (since its a singlet).

In certain cases (4.19) takes a relatively manageable form. One example is for $Y_{A}= \pm 1$ and $Y_{B}= \pm 2$, which is equivalent to the solution discussed in [42-45]. In this case, one can either require $Y_{A, L}^{\prime}=-1$ with $Y_{A, R}^{\prime}$ arbitrary or $Y_{A, R}^{\prime}=1$ with $Y_{A, L}^{\prime}$ arbitrary. The other case is $Y_{A}= \pm 7$ and $Y_{B}= \pm 10$, in which case one need only satisfy

$$
2 Y_{A, L}^{\prime}-3 Y_{A, R}^{\prime}+5=0 \quad \text { or } \quad 3 Y_{A, L}^{\prime}-2 Y_{A, R}^{\prime}+5=0
$$

to obtain acceptable solutions. In addition to these 'regular' solutions with a new $\mathrm{SU}(2)$ doublet fermion, we find 26 other 'exceptional' solutions that occur in 13 mirror pairs with $Y_{A, L}^{\prime} \leftrightarrow-Y_{A, R}^{\prime}$ that have $Y_{A / B} \leq 10$ and $Y_{A, L / R}^{\prime}= \pm p / q$ with $p, q \leq 10$. The simplest of these is

$$
\left(Y_{A, L}^{\prime}, Y_{A, R}^{\prime}, Y_{A}, Y_{B}\right)=\left(1, \frac{2}{3}, 3,2\right)
$$

which is accompanied by its mirror solution with $Y_{A, L}^{\prime} \leftrightarrow-Y_{A, R}^{\prime}$.

In addition to $Y_{\chi, L}^{\prime}=1$ by definition, the benchmark solution (4.21) has $Y_{l}^{\prime}=Y_{e}^{\prime}=0$ by construction, and hence

$$
Y_{q}^{\prime}=Y_{u}^{\prime}=Y_{d}^{\prime}, \quad Y_{H}^{\prime}=0
$$

where $Y_{q}^{\prime}$ is fixed by (4.15) and the values of $Y_{\chi, R}^{\prime}, Y_{B, L / R}^{\prime}$ are fixed by (4.16), (4.17) and (4.18)

$$
Y_{q}^{\prime}=-\frac{1}{27}, \quad Y_{\chi, R}^{\prime}=0, \quad Y_{B, L}^{\prime}=-\frac{1}{3}, \quad Y_{B, R}^{\prime}=\frac{4}{3} .
$$

We note that this solution admits a small quark charge relative to the DM charge, implying good complementarity between dijet and missing energy searches at the LHC. 
Finally, we consider the possibilities when $A$ is in the adjoint (triplet) representation of $\mathrm{SU}(2)_{W}$. In this case, the first four anomaly-cancellation conditions above are modified to

$$
\begin{aligned}
Y_{q}^{\prime} & =-\frac{4}{9}\left(Y_{A, L}^{\prime}-Y_{A, R}^{\prime}\right), \\
Y_{\chi, R}^{\prime} & =3 Y_{A, L}^{\prime}-3 Y_{A, R}^{\prime}+Y_{B, L}^{\prime}-Y_{B, R}^{\prime}+Y_{\chi, L}^{\prime}, \\
Y_{B, L}^{\prime} & =\frac{\left(3 Y_{A} Y_{B}^{3}+\left(3 Y_{A}^{2}+8\right)^{2}\right) Y_{A, R}^{\prime}-\left(\left(3 Y_{A}^{2}+8\right)^{2}-3 Y_{A} Y_{B}^{3}\right) Y_{A, L}^{\prime}}{2\left(3 Y_{A}^{2}+8\right) Y_{B}^{2}}, \\
Y_{B, R}^{\prime} & =\frac{\left(3 Y_{A} Y_{B}^{3}+\left(3 Y_{A}^{2}+8\right)^{2}\right) Y_{A, L}^{\prime}-\left(\left(3 Y_{A}^{2}+8\right)^{2}-3 Y_{A} Y_{B}^{3}\right) Y_{A, R}^{\prime}}{2\left(3 Y_{A}^{2}+8\right) Y_{B}^{2}},
\end{aligned}
$$

and the $\mathrm{U}(1)^{3}$ anomaly equation becomes

$$
\begin{aligned}
- & \frac{1}{8\left(3 Y_{A}^{2}+8\right)^{3} Y_{B}^{6}}\left[-24\left(3 Y_{A}^{2}+8\right)^{3} Y_{B}^{6}\left(\left(Y^{\prime}\right)_{A, L}^{3}-\left(Y^{\prime}\right)_{A, R}^{3}\right)\right. \\
& +8\left(3 Y_{A}^{2}+8\right)^{3}\left(Y_{B}^{2}\left(3 Y_{A, L}^{\prime}-3 Y_{A, R}^{\prime}+1\right)-\left(3 Y_{A}^{2}+8\right)\left(Y_{A, L}^{\prime}-Y_{A, R}^{\prime}\right)\right)^{3} \\
& +\left(\left(3 Y_{A} Y_{B}^{3}+\left(3 Y_{A}^{2}+8\right)^{2}\right) Y_{A, L}^{\prime}-\left(\left(3 Y_{A}^{2}+8\right)^{2}-3 Y_{A} Y_{B}^{3}\right) Y_{A, R}^{\prime}\right)^{3} \\
& +\left(\left(\left(3 Y_{A}^{2}+8\right)^{2}-3 Y_{A} Y_{B}^{3}\right) Y_{A, L}^{\prime}-\left(3 Y_{A} Y_{B}^{3}+\left(3 Y_{A}^{2}+8\right)^{2}\right) Y_{A, R}^{\prime}\right)^{3} \\
& \left.-8\left(3 Y_{A}^{2}+8\right)^{3} Y_{B}^{6}\right]=0 .
\end{aligned}
$$

As before we have the symmetry $Y_{A / B} \rightarrow-Y_{A / B}$. Requiring that $Y_{A}$ and $Y_{B}$ are even so as to obtain integer electric charges. we identify a set of solutions defined by $Y_{A}=0$ and $Y_{B}= \pm 2$, which satisfy

$$
Y_{A, R}^{\prime}=\frac{1+Y_{A, L}^{\prime}}{1+3 Y_{A, L}^{\prime}}
$$

In addition to $Y_{\chi, L}^{\prime}=1$ by definition, $Y_{A}=0$ and $Y_{B}= \pm 2$, and $Y_{A, L}^{\prime}$ as a free parameter that determines $Y_{A, R}^{\prime}$ via (4.30), this benchmark solution again has $Y_{l}^{\prime}=Y_{e}^{\prime}=0$ by construction and the conditions (4.22) are also obeyed, where $Y_{q}^{\prime}$ is fixed by (4.24), and the values of $Y_{\chi, R}^{\prime}, Y_{B, L / R}^{\prime}$ are fixed in this case by (4.25), (4.26) and (4.27). Choosing the positive solution $Y_{B}=2$, this relates the other charges:

$$
\begin{aligned}
Y_{q}^{\prime} & =\frac{4-12 Y_{A, L}^{\prime 2}}{9+27 Y_{A, L}^{\prime}} \\
Y_{\chi, R}^{\prime} & =\frac{3 Y_{A, L}^{\prime}\left(1+Y_{A, L}^{\prime}\right)}{1+3 Y_{A, L}^{\prime}} \\
Y_{B, L}^{\prime} & =\frac{1-3 Y_{A, L}^{\prime 2}}{1+3 Y_{A, L}^{\prime}} \\
Y_{B, R}^{\prime} & =-Y_{B, L}^{\prime}
\end{aligned}
$$

Picking a specific benchmark with $Y_{A, L}^{\prime}=1$, for example:

$$
Y_{A, R}^{\prime}=\frac{1}{2}, \quad Y_{q}^{\prime}=-\frac{2}{9}, \quad Y_{\chi, R}^{\prime}=\frac{3}{2}, \quad Y_{B, L}^{\prime}=-\frac{1}{2}, \quad Y_{B, R}^{\prime}=\frac{1}{2}
$$


As in the fundamental case, there are also 'exceptional' solutions not falling into the class described above. We find 28 such solutions with $Y_{A / B} \leq 10$ and $Y_{A, L / R}^{\prime}= \pm p / q$ with $p, q \leq 10$, occurring in 14 mirror pairs with $Y_{A, L}^{\prime} \leftrightarrow-Y_{A, R}^{\prime}$. The simplest of these is

$$
\left(Y_{A, L}^{\prime}, Y_{A, R}^{\prime}, Y_{A}, Y_{B}\right)=\left(-1,-\frac{3}{2}, 2,2\right)
$$

which is accompanied by its mirror solution.

Examining the gauge eigenstates, we find no solutions with an axial DM particle $Y_{\chi, L}^{\prime}=$ $-Y_{\chi, R}^{\prime}$ in this section. Therefore, ignoring the possible effects of mixing, we expect strong direct detection bounds to be relevant. However, based on our results in section 3, we expect that adding two SM-singlet dark fermions would allow an anomaly-free theory to exist in which one of the dark sector particles has an axial coupling.

As in the two-dark-fermion case studied in section 3, the interaction eigenstates $(A, B, \chi)$ in the models studied in this section will in general mix via a combination of 'Majorana' and 'Dirac' entries in the mass matrix, that are model-dependent. We do not discuss any details here, but note that many of the remarks made in section 3 apply here also: the mixing may give the lightest mass eigenstate (the DM particle) an admixture of vector-like coupling, which would vanish in the degenerate limit in which it was much lighter than the other mass eigenstates, and the heavier mass eigenstates would, in general, decay via off-diagonal $Z^{\prime}$ couplings into lighter mass eigenstates by emitting SM $\bar{f} f$ pairs. Finally we note that ,if the $\chi$ state mixes with a neutral component of $A$ or $B$, then a coupling to the SM $Z$ boson would be generated. Such a coupling is very heavily constrained, see, e.g., [63], putting pressure on the viability of $\chi$ as a DM candidate in such a case.

\section{Summary}

As we have seen in this paper, the cancellation of anomalies is a non-trivial constraint on SDMMs with a spin-one mediator boson $Z^{\prime}$. Our analysis has led us to consider three classes of models:

One exotic fermion. If the SM is supplemented by a single new fermion, a DM particle that is a singlet of the SM gauge group, the $Z^{\prime}$ cannot be leptophobic unless it also decouples from quarks. A benchmark model in this class is specified at the end of section 2, see (2.11). This model contains a single vector-like fermionic DM candidate which does not contribute to any anomalies - the assigned charges of the standard model fields alone cancel all anomalies. As such, this model is the Y-sequential model $[33,36]$ with the addition of a DM candidate. The relative coupling of the $Z^{\prime}$ to quarks and leptons is fixed and comparable, meaning that LHC dilepton bounds would rule out much of the parameter space. Moreover, to the extent that the DM particle has non-vanishing SM couplings, they must be vectorial, meaning that the cross section for scattering off a nucleus would not be velocity suppressed and would also be coherently enhanced. Therefore an SDMM with just a DM fermion and a $Z^{\prime}$ is very strongly constrained by LHC searches [54, 55] and direct DM scattering experiments $[56,57]$. 
Axial dark matter. If the DM particle is to have a purely axial $\mathrm{U}(1)^{\prime}$ coupling, which would diminish the impact of the DM scattering experiments $[56,57]$, then it must be accompanied by at least one other new singlet fermion. However, the $\mathrm{U}(1)^{\prime}$ charges of the SM leptons still do not vanish if there is a single such fermion, or several with identical charges. Thus, the $Z^{\prime}$ in such a model would still be subject to strong LHC constraints $[54,55]$. A benchmark model in this class is specified at the end of section 3, see (3.8) and (3.10).

Leptophobic models. We find several anomaly free leptophobic models only if the DM fermion is accompanied by at least two other new fermions with non-identical charges, at least one of which is a non-singlet under the SM gauge group. One of these models is the model with a baryonic DM particle presented in [42-45]. These models may be subject to constraints from LHC searches for new fermions with non-trivial SM quantum numbers that would need to be considered in assessing the parameter spaces of such models. A benchmark model with a new $\mathrm{SU}(2)_{W}$ doublet fermion is specified in (4.21) and (4.22), and one with a new $\mathrm{SU}(2)_{W}$ triplet fermion is specified in (4.30).

Beyond the specific models presented here, we re-emphasize the general point that proponents of SDMMs should ensure that they implement the anomaly-cancellation constraints. The bad news is that the resulting models may not be so simple, but the good news is that anomaly cancellation can relate the SM and DM couplings of the $Z^{\prime}$ and furthermore the additional fermions may have novel experimental signatures.

\section{Acknowledgments}

The work of JE and MF was supported partly by the STFC Grant ST/L000326/1. MF and PT are funded by the European Research Council under the European Union's Horizon 2020 programme (ERC Grant Agreement no. 648680 DARKHORIZONS).

Open Access. This article is distributed under the terms of the Creative Commons Attribution License (CC-BY 4.0), which permits any use, distribution and reproduction in any medium, provided the original author(s) and source are credited.

\section{References}

[1] F. Zwicky, Die Rotverschiebung von extragalaktischen Nebeln, Helv. Phys. Acta 6 (1933) 110 [INSPIRE].

[2] F. Zwicky, On the Masses of Nebulae and of Clusters of Nebulae, Astrophys. J. 86 (1937) 217 [INSPIRE].

[3] V.C. Rubin and W.K. Ford, Jr., Rotation of the Andromeda Nebula from a Spectroscopic Survey of Emission Regions, Astrophys. J. 159 (1970) 379 [INSPIRE].

[4] V.C. Rubin, N. Thonnard and W.K. Ford, Jr., Rotational properties of 21 SC galaxies with a large range of luminosities and radii, from $N G C 4605 / R=4 \mathrm{kpc} /$ to UGC2885/R=122 kpc/, Astrophys. J. 238 (1980) 471 [INSPIRE].

[5] Y. Sofue and V. Rubin, Rotation curves of spiral galaxies, Ann. Rev. Astron. Astrophys. 39 (2001) 137 [astro-ph/0010594] [INSPIRE]. 
[6] J.P. Ostriker, P.J.E. Peebles and A. Yahil, The size and mass of galaxies and the mass of the universe, Astrophys. J. 193 (1974) L1 [INSPIRE].

[7] D. Clowe et al., A direct empirical proof of the existence of dark matter, Astrophys. J. 648 (2006) L109 [astro-ph/0608407] [INSPIRE].

[8] Planck collaboration, P.A.R. Ade et al., Planck 2015 results. XIII. Cosmological parameters, Astron. Astrophys. 594 (2016) A13 [arXiv:1502.01589] [INSPIRE].

[9] M.G. Walker, Dark Matter in the Milky Way's Dwarf Spheroidal Satellites, in Planets, Stars and Stellar Systems. Volume 5: Galactic Structure and Stellar Populations, Springer Verlag, Heidelberg Germany (2013) [arXiv:1205.0311].

[10] G. Bertone and D. Hooper, A History of Dark Matter, Submitted to: Rev. Mod. Phys. (2016) [arXiv: 1605.04909] [INSPIRE].

[11] G. Bertone, D. Hooper and J. Silk, Particle dark matter: Evidence, candidates and constraints, Phys. Rept. 405 (2005) 279 [hep-ph/0404175] [INSPIRE].

[12] J.R. Ellis, J.S. Hagelin, D.V. Nanopoulos, K.A. Olive and M. Srednicki, Supersymmetric Relics from the Big Bang, Nucl. Phys. B 238 (1984) 453 [INSPIRE].

[13] H. Goldberg, Constraint on the Photino Mass from Cosmology, Phys. Rev. Lett. 50 (1983) 1419 [Erratum ibid. 103 (2009) 099905] [INSPIRE].

[14] M. Beltrán, D. Hooper, E.W. Kolb and Z.C. Krusberg, Deducing the nature of dark matter from direct and indirect detection experiments in the absence of collider signatures of new physics, Phys. Rev. D 80 (2009) 043509 [arXiv:0808.3384] [INSPIRE].

[15] M. Beltrán, D. Hooper, E.W. Kolb, Z.A.C. Krusberg and T.M.P. Tait, Maverick dark matter at colliders, JHEP 09 (2010) 037 [arXiv: 1002.4137] [INSPIRE].

[16] Y. Bai, P.J. Fox and R. Harnik, The Tevatron at the Frontier of Dark Matter Direct Detection, JHEP 12 (2010) 048 [arXiv:1005.3797] [INSPIRE].

[17] I.M. Shoemaker and L. Vecchi, Unitarity and Monojet Bounds on Models for DAMA, CoGeNT and CRESST-II, Phys. Rev. D 86 (2012) 015023 [arXiv:1112.5457] [INSPIRE].

[18] P.J. Fox, R. Harnik, R. Primulando and C.-T. Yu, Taking a Razor to Dark Matter Parameter Space at the LHC, Phys. Rev. D 86 (2012) 015010 [arXiv:1203.1662] [InSPIRE].

[19] G. Busoni, A. De Simone, E. Morgante and A. Riotto, On the Validity of the Effective Field Theory for Dark Matter Searches at the LHC, Phys. Lett. B 728 (2014) 412 [arXiv: 1307.2253] [INSPIRE].

[20] G. Busoni, A. De Simone, J. Gramling, E. Morgante and A. Riotto, On the Validity of the Effective Field Theory for Dark Matter Searches at the LHC, Part II: Complete Analysis for the s-channel, JCAP 06 (2014) 060 [arXiv:1402.1275] [INSPIRE].

[21] O. Buchmueller, M.J. Dolan and C. McCabe, Beyond Effective Field Theory for Dark Matter Searches at the LHC, JHEP 01 (2014) 025 [arXiv: 1308.6799] [INSPIRE].

[22] O. Buchmueller, S.A. Malik, C. McCabe and B. Penning, Constraining Dark Matter Interactions with Pseudoscalar and Scalar Mediators Using Collider Searches for Multijets plus Missing Transverse Energy, Phys. Rev. Lett. 115 (2015) 181802 [arXiv:1505.07826] [INSPIRE]. 
[23] S.A. Malik et al., Interplay and Characterization of Dark Matter Searches at Colliders and in Direct Detection Experiments, Phys. Dark Univ. 9-10 (2015) 51 [arXiv:1409.4075] [INSPIRE].

[24] J. Abdallah et al., Simplified Models for Dark Matter Searches at the LHC, Phys. Dark Univ. 9-10 (2015) 8 [arXiv:1506.03116] [INSPIRE].

[25] M. Bauer et al., Towards the next generation of simplified Dark Matter models, White Paper from 2016 Brainstorming Workshop held at Imperial College London, London U.K. (2016) [arXiv: 1607.06680].

[26] G. Arcadi, Y. Mambrini, M.H.G. Tytgat and B. Zaldivar, Invisible $Z^{\prime}$ and dark matter: LHC vs LUX constraints, JHEP 03 (2014) 134 [arXiv: 1401.0221] [INSPIRE].

[27] D. Abercrombie et al., Dark Matter Benchmark Models for Early LHC Run-2 Searches: Report of the ATLAS/CMS Dark Matter Forum, arXiv:1507.00966 [INSPIRE].

[28] G. Busoni et al., Recommendations on presenting LHC searches for missing transverse energy signals using simplified s-channel models of dark matter, arXiv:1603.04156 [INSPIRE].

[29] M. Fairbairn, J. Heal, F. Kahlhoefer and P. Tunney, Constraints on $Z^{\prime}$ models from LHC dijet searches and implications for dark matter, JHEP 09 (2016) 018 [arXiv:1605.07940] [INSPIRE].

[30] T. Jacques, A. Katz, E. Morgante, D. Racco, M. Rameez and A. Riotto, Complementarity of DM searches in a consistent simplified model: the case of $Z^{\prime}$, JHEP 10 (2016) 071 [arXiv: 1605.06513] [INSPIRE].

[31] F. Kahlhoefer, K. Schmidt-Hoberg, T. Schwetz and S. Vogl, Implications of unitarity and gauge invariance for simplified dark matter models, JHEP 02 (2016) 016 [arXiv: 1510.02110] [INSPIRE].

[32] M.S. Chanowitz, J.R. Ellis and M.K. Gaillard, The Price of Natural Flavor Conservation in Neutral Weak Interactions, Nucl. Phys. B 128 (1977) 506 [INSPIRE].

[33] A. Ekstedt, R. Enberg, G. Ingelman, J. Löfgren and T. Mandal, Constraining minimal anomaly free U(1) extensions of the Standard Model, JHEP 11 (2016) 071 [arXiv: 1605. 04855] [INSPIRE].

[34] S.M. Barr, B. Bednarz and C. Benesh, Anomaly Constraints and New U(1) Gauge Bosons, Phys. Rev. D 34 (1986) 235 [inSPIRE].

[35] J. Erler and P. Langacker, Constraints on extended neutral gauge structures, Phys. Lett. B 456 (1999) 68 [hep-ph/9903476] [INSPIRE].

[36] T. Appelquist, B.A. Dobrescu and A.R. Hopper, Nonexotic neutral gauge bosons, Phys. Rev. D 68 (2003) 035012 [hep-ph/0212073] [INSPIRE].

[37] M. Carena, A. Daleo, B.A. Dobrescu and T.M.P. Tait, $Z^{\prime}$ gauge bosons at the Tevatron, Phys. Rev. D 70 (2004) 093009 [hep-ph/0408098] [INSPIRE].

[38] P. Batra, B.A. Dobrescu and D. Spivak, Anomaly-free sets of fermions, J. Math. Phys. 47 (2006) 082301 [hep-ph/0510181] [INSPIRE].

[39] D.E. Morrissey and J.D. Wells, The Tension between gauge coupling unification, the Higgs boson mass and a gauge-breaking origin of the supersymmetric mu-term, Phys. Rev. D 74 (2006) 015008 [hep-ph/0512019] [INSPIRE]. 
[40] C.-W. Chiang, N.G. Deshpande and J. Jiang, Flavor changing effects in family nonuniversal $Z^{\prime}$ models, JHEP 08 (2006) 075 [hep-ph/0606122] [INSPIRE].

[41] P. Langacker, The Physics of Heavy Z' Gauge Bosons, Rev. Mod. Phys. 81 (2009) 1199 [arXiv:0801.1345] [INSPIRE].

[42] P. Fileviez Perez, S. Ohmer and H.H. Patel, Minimal Theory for Lepto-Baryons, Phys. Lett. B 735 (2014) 283 [arXiv: 1403.8029] [INSPIRE].

[43] M. Duerr, P. Fileviez Perez and M.B. Wise, Gauge Theory for Baryon and Lepton Numbers with Leptoquarks, Phys. Rev. Lett. 110 (2013) 231801 [arXiv:1304.0576] [inSPIRE].

[44] M. Duerr and P. Fileviez Perez, Theory for Baryon Number and Dark Matter at the LHC, Phys. Rev. D 91 (2015) 095001 [arXiv: 1409.8165] [InSPIRE].

[45] P. Fileviez Perez and M.B. Wise, Baryon and lepton number as local gauge symmetries, Phys. Rev. D 82 (2010) 011901 [Erratum ibid. D 82 (2010) 079901] [arXiv:1002.1754] [INSPIRE].

[46] A. Ismail, W.-Y. Keung, K.-H. Tsao and J. Unwin, Axial vector $Z^{\prime}$ and anomaly cancellation, Nucl. Phys. B 918 (2017) 220 [arXiv: 1609.02188] [INSPIRE].

[47] D. Hooper, $Z^{\prime}$ Mediated Dark Matter Models for the Galactic Center Gamma-Ray Excess, Phys. Rev. D 91 (2015) 035025 [arXiv:1411.4079] [INSPIRE].

[48] B.A. Dobrescu and C. Frugiuele, Hidden GeV-scale interactions of quarks, Phys. Rev. Lett. 113 (2014) 061801 [arXiv:1404.3947] [INSPIRE].

[49] E. Dudas, L. Heurtier, Y. Mambrini and B. Zaldivar, Extra U(1), effective operators, anomalies and dark matter, JHEP 11 (2013) 083 [arXiv:1307.0005] [INSPIRE].

[50] W. Wang and Z.-L. Han, Radiative linear seesaw model, dark matter and $\mathrm{U}(1)_{B-L}$, Phys. Rev. D 92 (2015) 095001 [arXiv: 1508.00706] [inSPIRE].

[51] J.M. Berryman, A. de Gouvêa, D. Hernández and K.J. Kelly, Imperfect mirror copies of the standard model, Phys. Rev. D 94 (2016) 035009 [arXiv: 1605.03610] [INSPIRE].

[52] A. de Gouvêa and D. Hernández, New Chiral Fermions, a New Gauge Interaction, Dirac Neutrinos and Dark Matter, JHEP 10 (2015) 046 [arXiv:1507.00916] [INSPIRE].

[53] P. Langacker, R.W. Robinett and J.L. Rosner, New Heavy Gauge Bosons in $p$ p and $p \bar{p}$ Collisions, Phys. Rev. D 30 (1984) 1470 [InSPIRE].

[54] CMS collaboration, Search for a high-mass resonance decaying into a dilepton final state in $13 \mathrm{fb}^{-1}$ of pp collisions at $\sqrt{\mathrm{s}}=13 \mathrm{TeV}$, CMS-PAS-EXO-16-031 (2016), https://cds.cern.ch/record/2205764/files/EXO-16-031-pas.pdf.

[55] ATLAS collaboration, Search for new high-mass phenomena in the dilepton final state using $36.1 \mathrm{fb}^{-1}$ of proton-proton collision data at $\sqrt{s}=13 \mathrm{TeV}$ with the ATLAS detector, ATLAS-CONF-2017-027 (2017), https://cds.cern.ch/record/2259039/files/ATLAS-CONF-2017-027.pdf.

[56] PandaX-II collaboration, A. Tan et al., Dark Matter Results from First 98.7 Days of Data from the PandaX-II Experiment, Phys. Rev. Lett. 117 (2016) 121303 [arXiv:1607.07400] [INSPIRE].

[57] LUX collaboration, D.S. Akerib et al., Results from a search for dark matter in the complete LUX exposure, Phys. Rev. Lett. 118 (2017) 021303 [arXiv: 1608.07648] [INSPIRE]. 
[58] J. Kumar and D. Marfatia, Matrix element analyses of dark matter scattering and annihilation, Phys. Rev. D 88 (2013) 014035 [arXiv:1305.1611] [INSPIRE].

[59] F. D'Eramo, B.J. Kavanagh and P. Panci, You can hide but you have to run: direct detection with vector mediators, JHEP 08 (2016) 111 [arXiv:1605.04917] [INSPIRE].

[60] S.L. Glashow and S. Weinberg, Natural Conservation Laws for Neutral Currents, Phys. Rev. D 15 (1977) 1958 [INSPIRE].

[61] E.A. Paschos, Diagonal Neutral Currents, Phys. Rev. D 15 (1977) 1966 [inSPIRE].

[62] M.L. Perl, E.R. Lee and D. Loomba, Searches for fractionally charged particles, Ann. Rev. Nucl. Part. Sci. 59 (2009) 47 [InSPIRE].

[63] M. Escudero, A. Berlin, D. Hooper and M.-X. Lin, Toward (Finally!) Ruling Out Z and Higgs Mediated Dark Matter Models, JCAP 12 (2016) 029 [arXiv:1609.09079] [InSPIRE]. 